Relations industrielles

Industrial Relations

\title{
Santé et sécurité au travail, Gouvernement du Québec, Québec, Éditeur Officiel du Québec, 1978, 289 pp.
}

Alain Vinet

Volume 34, numéro 2, 1979

URI : https://id.erudit.org/iderudit/028973ar

DOI : https://doi.org/10.7202/028973ar

Aller au sommaire du numéro

Éditeur(s)

Département des relations industrielles de l'Université Laval

ISSN

0034-379X (imprimé)

1703-8138 (numérique)

Découvrir la revue

Citer ce compte rendu

Vinet, A. (1979). Compte rendu de [Santé et sécurité au travail, Gouvernement du Québec, Québec, Éditeur Officiel du Québec, 1978, 289 pp.] Relations

industrielles / Industrial Relations, 34(2), 383-385.

https://doi.org/10.7202/028973ar

Tous droits réservés (C) Département des relations industrielles de l'Université Laval, 1979
Ce document est protégé par la loi sur le droit d'auteur. L'utilisation des services d'Érudit (y compris la reproduction) est assujettie à sa politique d'utilisation que vous pouvez consulter en ligne.

https://apropos.erudit.org/fr/usagers/politique-dutilisation/ 
production par travailleur soulève certains problèmes. La comparaison de la productivité d'une province avec celle du Canada suppose implicitement que la moyenne canadienne représente la norme de comparaison tout indiquée de la productivité des diverses provinces. Si, par exemple, dans une province donnée, la proportion de l'emploi dans une industrie à forte productivité est au-dessus de la moyenne, ce serait le signe d'une structure industrielle appropriée ou, vice versa, si une industrie à faible productivité jouit d'une répartition de l'emploi supérieure à la moyenne nationale, cela indiquerait une mauvaise structure industrielle. En se basant sur la théorie, il est impossible de soutenir pareille déduction.

Prenons, par hypothèse, le cas d'une province généreusement dotée en terres agricoles de haute qualité qui lui confèrent un avantage naturel dans le domaine de l'agriculture. Serait-il préférable pour cette province de ramener sa répartition de l'emploi dans le secteur de l'agriculture au niveau de la moyenne nationale ou aurait-elle avantage à exploiter à fond cette richesse naturelle? Si l'on s'en tient à la théorie, la répartition de l'emploi atteindrait une valeur optimale si le rapport du produit de recettes marginales et du coût marginal de la main-d'oeuvre était le même dans toutes les industries de cette province. Selon la dotation en ressources, ce rapport pourrait cependant varier fortement d'une province à l'autre et, partant, la répartition optimale de l'emploi au niveau provincial.

On pourrait donc prétendre que la structure industrielle, mesurée en termes de répartition de l'emploi dans les différentes industries, ne peut être réputée bonne ou mauvaise en regard des données concernant le Canada, mais uniquement en comparaison d'une norme qui rend compte des écarts interrégionaux en matière de productivité marginale et de coûts. L'estimation empirique d'une telle norme de la répartition optimale des ressources n'est pas impensable, mais l'expérience a démontré que les résultats tirés d'une démarche semblable ne sont peut- être pas assez «solides» pour mener à des estimations sûres sans le recours à des essais multiples. Pour simplifier le processus, on a retenu la moyenne canadienne comme norme de comparaison».

Pour conclure, les études sur la productivité peuvent envisager deux questions, le "comment» et le "pourquoi». Ce travail se limite au "comment» puisqu'il n'est qu'une forme de comptabilisation ou de décomposition d'une mesure statistique. Il ne répond nullement au «pourquoi» des écarts de la productivité. Même s'il est utile de savoir le "comment», ce n'est que par l'identification du «pourquoi» qu'il est possible d'être prescriptif. C'est là qu'est tout le rôle de l'analyse économique.

Gérard BÉLANGER

Université Laval

Santé et sécurité au travail, Gouvernement du Québec, Québec, Editeur Officiel du Québec, 1978, 289 pp.

Ce livre blanc, très attendu, concernant la santé et la sécurité des travailleurs, a déjà fait couler beaucoup d'encre. Commentaires favorables et critiques acerbes se sont multipliés depuis sa parution. Le Ministre responsable du dossier prépare présentement une législation qui devrait concrétiser les intentions du gouvernement à ce sujet mais également introduire des amendements substantiels aux politiques énoncées dans le Livre blanc. Le débat demeure donc largement ouvert sur le régime proposé par le gouvernement pour assurer la sécurité des travailleurs et protéger leur santé.

Mais l'ampleur même de ce débat et l'écho qu'il suscite dans les média d'information contribuent à nous faire oublier que la majeure partie de ce document gouvernemental est consacrée à une description rigoureuse de la situation qui prévaut actuellement au Québec en matière de santé et de sécurité au travail: fréquence et gravité des accidents du travail et des maladies professionnelles 
selon les secteurs d'activité économique; identification des risques auxquels s'exposent les travailleurs québécois; décompte des décès, des invalidités permanentes et des journées de travail perdues chaque année; identification des coûts reliés aux accidents du travail et aux maladies professionnelles. Et ce n'est pas tout. Usant d'une écriture aisément accessible au profane, les auteurs décrivent le labyrinthe juridique et administratif qui encadre les questions de santé et de sécurité au travail, cernent les failles des mécanismes de contrôle et de prévention en vigueur, et critiquent le régime actuel d'indemnisation trop peu soucieux de la réadaptation sociale des accidentés du travail.

C'est donc d'un diagnostic global qu'il s'agit. Le tableau en paraît très sombre. Environ un travailleur sur huit est victime d'un accident ou d'une maladie provoquée par son travail chaque année; en 1977, on dénombre 265,000 accidents du travail; en 1976, 8,552 cas d'incapacité permanente et 245 décès; la même année, 7,782,000 journées de travail perdues. Mais l'intérêt du Livre blanc tient surtout au fait que ces chiffres sont analysés selon les secteurs d'activité économique, et tiennent compte dans chaque secteur du nombre d'accidents et de maladies par 100 travailleurs et du nombre moyen de journées d'absence. En quelques pages, on découvre une véritable mine de renseignements, parfois inédits. Cet effort de synthèse est remarquable, à partir de données disparates et dispersées. Une faiblesse cependant: la santé mentale des travailleurs à laquelle on consacre trois paragraphes, faute d'informations précises sans doute mais aussi parce que les problèmes de cet ordre, bien que sérieux et fréquents, sont moins visibles et moins spectaculaires.

Le même effort de synthèse et de vulgarisation caractérise les chapitres consacrés au cadre juridique et administratif, aux mécanismes de contrôle et de prévention et au fonctionnement du régime d'indemnisation. Le lecteur a sous la main des informations auparavant dispersées. Il est également en mesure d'identifier plusieurs failles importantes du régime actuel: le morcellement et la juxtapo- sition des juridictions qui entraînent des dédoublements coûteux et une inefficacité certaine; l'absence de rôle dévolu au travailleur dans les questions de santé et de sécurité; le peu d'activités destinées à informer et à former le travailleur dans son milieu de travail; l'insuffisance des services de santé à la disposition des travailleurs; l'absence d'une politique de recherche et l'absence d'une procédure systématique de cueillette d'informations sur les facteurs de risque auxquels sont exposés les travailleurs québécois.

Pour remédier à cette situation, pour assurer la sécurité des travailleurs et protéger leur santé, le gouvernement propose un nouveau régime dont les principales dispositions ont été longuement commentées dans les média. Point n'est besoin de les résumer de nouveau ni de souligner qu'elles ne font pas toutes l'unanimité des milieux concernés. Le lecteur désireux d'établir son opinion trouvera profit à comparer la seconde partie du Livre blanc qui établit la position gouvernementale et le document de travail de la FTQ, intitulé La FTQ et le Livre blarıc, et diffusé lors du Colloque sur la santé et la sécurité au travail tenu par cette centrale syndicale en novembre dernier.

Les dispositions concernant le droit individuel du travailleur de cesser un travail dangereux sont particulièrement controversées. Elles prennent même figure de symbole dans un débat socio-politique où les problèmes de santé et de sécurité au travail posent la question plus vaste de l'évolution des relations de pouvoir dans l'entreprise. Où se situeront désormais les points d'équilibre entre le traditionnel droit de gérance de l'employeur et le droit revendiqué par les syndicats d'enquêter partout, en tout temps, sur tout sujet relatif à la santé et à la sćcurité au travail et, s'il y a lieu, de cesser un travail dangereux au nom des travailleurs en cause? Quelles seront les fonctions respectives de la réglementation et de la négociation dans le règlement des questions de santé et de sécurité au travail? Comment seront partagés les rôles et les moyens mis à la disposition des organismes publics, des employeurs et des syndicats dans le but 
d'assurer la formation des travailleurs? Quelles garanties de protection sera-t-on en mesure d'offrir aux travailleurs non syndiqués?

Ces questions et de nombreuses autres alimenteront encore longtemps le débat. Des amendements à la politique énoncée sont prévus. Par conséquent, certains aspects de cette seconde partie du Libre blanc sont déjà périmés. Ce qui n'enlève rien à la valeur et à la portée du diagnostic établi dans ce document et ne remet pas en cause les objectifs fondamentaux de la réforme.

\section{Alain VINET}

Université Laval

\section{Retour sur le régime caché d'assistance socia- le, Ottawa, Rapport du Conseil canadien du bien-être social, mars 1979, 37 pp.}

À une période où il semble politiquement nécessaire de ralentir la croissance des dépenses gouvernementales et d'alléger le fardeau fiscal des éléments et des groupes productifs de la société, à en juger par le succès de la "proposition 13» de la Californie ou encore par la prudence des budgets tant fédéral que provinciaux pour 1979-80, ce nouveau Rapport du Conseil canadien du bien-être social arrive, si l'on peut dire, à point.

En effet, dans tout le débat entourant la relation entre la fiscalité et la croissance économique, on a subtilement glissé au second plan celui sur la redistribution du revenu national et cela de deux façons: d'une part, en abordant les modifications aux divers programmes affectant la redistribution du revenu et en termes absolus plutôt que relatifs (voire même réels) mais plus subtilement encore en termes de "dépenses directes» plutôt qu'en termes de dépenses globales. C'est sur le deuxième «discours caché» que le CCBES attire l'attention, s'inspirant en cela d'un intérêt marqué depuis plusieurs années chez nos voisins du Sud pour cette question vu la complexité et l'importance des régimes dits de "déductions» et "d'exemptions» que comporte la fiscalité américaine.

L'idée est simple. Toutes politiques de redistribution du revenu opère à partir des recettes (entrées fiscales) ou impôts et des dépenses. Or, il existe deux types de dépenses: directes et cachées, et chaque type affecte la redistribution du revenu. Les dépenses directes sont comptabilisées dans les comptes publics, pas les dépenses cachées. Par dépenses cachées, on entend ces argents redistribués simultanément au moment de la perception des impôts via des déductions et exemptions fiscales de toutes sortes. En effet, tout contribuable est soumis à un régime universel de base d'imposition mais peut recevoir de l'État des sommes sous formes d'allègement fiscal (non universel ou bien indépendant de la progressivité du régime ou de transferts après impôt. Il s'agit toutefois de dépenses nonbudgétées, non comptabilisées, dont il est donc difficile d'en faire l'évaluation et d'en connaître les effets de distorsions réelles sur la distribution du revenu

L'étude du CCBES porte sur plus d'une vingtaine d'exemptions, déductions et crédits dans le régime d'impôt sur le revenu des particuliers. Une étude plus exhaustive incluerait l'ensemble du régime d'impôt mais même au seul niveau retenu ici, les observations du CCBES sur des «programmes cachés» tels les cotisations d'assurance-chômage, les régimes enregistrés de pension, d'épargne-retraite, d'épargne-logement, les exemptions pour personnes à charge, les déductions pour intérêts, dividendes, etc.

L'analyse présente plusieurs tableaux bidimensionnels où l'on trouve les taux annuels de progressions de ces dépenses de 1924 à 1976 ainsi que la distribution, par catégories de revenus (en déciles) des bénéficiaires de ces dépenses dans le cas de certains programmes.

Une analyse limitée mais fort utile pour aider à replacer le débat sur la redistribution en termes de "piastres et de cents".

Jacques MERCIER

Université Laval 